\title{
Application of high performance liquid chromatography - inductively coupled plasma mass spectrometry to the investigation of phytochelatin complexes and their role in heavy metal detoxification in plants
}

\author{
I. Leopold ${ }^{1}$, D. Günther ${ }^{2}$ and D. Neumann ${ }^{3}$ \\ ${ }^{1}$ Laboratory for Materials and Nuclear Processes, Paul Scherrer Institute, 5232 Villigen PSI, Switzerland \\ ${ }^{2}$ Institute of Isotope Geology and Mineral Resources, Swiss Institute of Technology Zürich, Sonneggstrasse 5, 8092 Zürich, Switzerland \\ ${ }^{3}$ Institute of Plant Biochemistry, Weinberg 3, 06120 Halle, Germany
}

The coupling of size-exclusion chromatography with ICP-MS is shown to be an attractive technique to study the binding of cadmium and copper by phytochelatins in plant cell suspension cultures incubated with these metals. Electrospray mass spectrometry enabled to identify the metal binding compounds: $(\gamma-\text { Glu-Cys })_{2}-$ Gly and $(\gamma \text {-Glu-Cys) })_{2}$.

\section{Heavy metal tolerance in plant systems}

Heavy metals (HMs) like cadmium, copper, zinc, lead, or mercury are environmental pollutants inducing physiological alterations in plants such as growth retardation, membrane damage, changes in enzyme activities or induction of oxidative stress when their concentrations exceed an element specific threshold. HM ions interact with $\mathrm{SH}$ - and histidyl groups of enzymes and membrane proteins, leading to inactivation of the proteins, cell damage and finally cell death [1]. However, a number of plant phenotypes with increased resistance to HM ions have been isolated from locations that are polluted with high concentrations of different metals. The physiological basis of the HM tolerance of these plants can be addressed either to a reduced uptake of HM ions into the plant cells or to the development of metal-sequestering mechanisms within different cellular compartments. Some of these detoxification mechanisms in HM exposed plants are metal binding to the cell walls, reduced transport across the membranes, active efflux, precipitation in vacuoles, 
synthesis of HM binding compounds such as peptides, proteins, organic acids or phenolic compounds $[2,3]$.

The storage of HMs inside the vacuoles presumes a translocation of the HM ions through the cytoplasm. It is widely accepted that HMs pass the cytoplasm in a chelated form. For Ni the binding to the imidazole nitrogen of histidine was shown [4]. The synthesis of phytochelatins (PCs) is a common response of plant cells upon exposure to HM ions. PCs are small peptides with the general structure $(\gamma \text {-Glu-Cys })_{n}-$ Gly $(n=2-11)$ that are able to chelate HMs due to the high cysteine content in the molecule. The synthesis of PC molecules with different chain length is induced by a wide variety of $\mathrm{HMs}$ (e.g. $\mathrm{Ag}, \mathrm{Bi}, \mathrm{Cd}, \mathrm{Cu}, \mathrm{Hg}, \mathrm{Pb}, \mathrm{Sn}$, $\mathrm{Zn}$ [5]), whereas the direct participation of PCs in the chelation of HM ions in plants has been verified for only few elements under in vivo and in vitro conditions. Very few data about the binding properties of the HM-PC-complexes are known, mainly due to the problem that all the crystallisation efforts have failed to date. In order to understand the role of these peptides for HM detoxification in plants and for the development of HM tolerant phenotypes a detailed knowledge of the metal-binding characteristics, such as binding affinity or binding stability is needed.

\section{Analysis of PC molecules and their HM binding complexes}

The separation of the dissociated HM-PC-complexes by a reversed phase HPLC with post-column derivatization [6] or fluorescent labelling of the free SH-groups [7] are commonly used methods for the qualitative and quantitative analysis of metal-free PC isoforms. Because the investigation of the binding properties and of the HM ions selectively bound to PC complexes is only possible under non-denaturing conditions, a time consuming combination of different chromatographic procedures (e.g. gel filtration and ion chromatography) and the subsequent determination of the HMs by element specific methods (e.g. AAS) in the separated fractions has been mostly applied.

In a complex biological matrix trace elements can be present in ionic form, as low or high molecular weight compounds or as organometal complexes. Element specific analytical methods (e.g. AAS, ICP AES or ICP MS) can only determine the total content of elements in a sample, but these techniques are not able to distinguish between different forms of an element, the so called "species", which are very important for understanding the biological function or the toxicity of an element. For the determination of the concentration of individual elemental species a separation step of each of these compounds is necessary prior to the analysis by AAS, ICP AES or ICP MS. Many of these off-line or online coupled separation and detection systems have been used in the past for speciation analysis in biological, environmental, and clinical research.

In the present study an on-line coupled high performance liquid chromatography - inductively coupled plasma mass spectrometry system (HPLC-ICP MS) was used for the investigation of the binding properties of HM-PC-complexes in order to characterise the role of these compounds for the $\mathrm{HM}$ detoxification in HM tolerant and non-tolerant plants.
Speciation techniques address only a fraction of the total metal content present in the samples, requiring highly sensitive analytical detectors. ICP MS was chosen because it has several attractive features compared with other analytical techniques: isotopic information is available, multielemental capability allows a high sample throughput, detection limits are superior to most conventional techniques with a wide linear dynamic range of the detector, and most of the elements in the periodic table can be detected.

Regarding the separation technique prior to the element specific detection, gas or liquid chromatography and capillary electrophoresis are the preferred methods because they are known as highly efficient for a wide variety of samples [8]. In principle, each chromatographic system can be used for species analysis; the selection of the separation method depends on the sample properties itself. In comparison to an off-line combination of the separation technique and the element specific detector the on-line coupling of both devices has the great advantage of a fast time resolved analysis with almost no sample lost or contamination because the separated compounds are directly transported to the excitation source and to the detector and are not collected in fractions and stored for subsequent analysis. Due to the fact that the separation of the components of interest from the sample matrix is a direct part of the analysis procedure itself, time consuming sample preparation steps such as purification and preconcentration can normally be avoided. A styrene-divinylbenzene-copolymer based size exclusion chromatographic column was selected for this study because of low interactions with metals during the separation process compared to silica gels and the possibility of working under isocratic conditions with low concentrated buffers and without any organic solvents in the mobile phase, which is very advantageous when coupling the HPLC to an ICP MS detector.

\section{Sample preparation}

One of the problems of speciation analysis in biological extracts is the development of a representative sampling procedure. Unfortunately, intensive sample pretreatment can significantly modify the yield of the different elemental species detected in the sample. In order to keep the number of preparation steps as low as possible a very simple extraction procedure was used for cell suspension cultures and whole plants.

Water cultures as well as cell suspension cultures of a HM tolerant plant, Silene vulgaris, and of tomato as a non-tolerant plant, incubated with different $\mathrm{HM}$ ion concentrations were used for the analysis of the soluble HM-binding compounds. In order to characterise the binding affinity and binding stability of HM-PC-complexes the plants were exposed with essential $\left(\mathrm{Cu}^{2+}, \mathrm{Zn}^{2+}\right)$ and non-essential $\left(\mathrm{Cd}^{2+}\right.$, $\mathrm{Pb}^{2+}$ ) HM ions in short- and long-term experiments. The cell suspension cultures were harvested by suction filtration, washed with $0.1 \mathrm{M}$ EDTA to remove extracellulary bound HM ions and immediately frozen in liquid nitrogen for storage. All sample preparation steps for cell cultures and whole plants were carried out in the same way as described elsewhere [9]. The most important points of the sample pretreatment are a low temperature, the addition of 2-mercaptoethanol to all extracts, and a $\mathrm{pH}$-value of about 8 in order 
to prevent the oxidation of the SH-groups in the samples and the dissociation of the HM-binding complexes which would occur at $\mathrm{pH} 6$ or lower. After the extraction procedure the samples were directly analysed without any cleanup or concentration step, minimising the potential risk of contamination. The separation conditions and the detailed operating parameters of the on-line coupled HPLC-ICP MS system are briefly summarised in table I.

\section{Results and discussion}

All parameters affecting the resolution of the HPLC separation, such as $\mathrm{pH}$ and chemical composition of the mobile phase, and their influence on the ICP mass spectrometric detection of the transient signals were investigated on a PC reference sample well characterised for the peptide composition. The metal concentration of these sample was unknown. The best analytical performance of the HPLC-ICP MS system was achieved at a $\mathrm{pH}$ value of 7, $10 \mathrm{mM}$ ammonium acetate as the mobile phase and a flow rate between 0.5 and $1.0 \mathrm{~mL} / \mathrm{min}$. A lower $\mathrm{pH}$ results in the displacement of the HM ions from the PC-complex; a higher concentration of the ammonium acetate leads to an increase of the background intensity for different elements (e.g. Zn) and therefore to a deterioration of the detection limits.

The analysis of extracts of Silene vulgaris and tomato cell suspension cultures shows a newly synthesised compound with a molecular weight of approximately $13000 \mathrm{Da}$ which can be observed in the UV chromatogram and was not detectable in the control plants (Fig. 1). The corresponding transient ICP MS signals of these extracts clearly indicate that this compound is a HM-binding complex with the applied HM ions in both cell suspension cultures (Fig. 2). Informations about the structure of the HM-binding complex are not available from the HPLC-ICP MS measurements. Therefore, the purified HM-binding compound of both cell

Table I. Chromatographic conditions and operating parameters of the HPLC-ICP MS system.

\section{HPLC}

\begin{tabular}{|c|c|}
\hline $\begin{array}{l}\text { Mobile phase } \\
\text { Flow rate } \\
\text { Death time between } \\
\text { UV and ICP-MS } \\
\text { detector } \\
\text { Injection volume }\end{array}$ & $\begin{array}{l}10 \mathrm{mM} \text { ammonium acetate, } \mathrm{pH} 7 \\
0.5-1.0 \mathrm{~mL} / \mathrm{min} \\
30-60 \mathrm{~s}\end{array}$ \\
\hline \multicolumn{2}{|l|}{ ICP-MS } \\
\hline $\begin{array}{l}\text { RF forward power } \\
\text { Reflected power } \\
\text { Cool gas flow } \\
\text { Auxiliary gas flow } \\
\text { Nebulizer gas flow } \\
\text { Data aquisition mode } \\
\text { Points per peak } \\
\text { Dwell time }\end{array}$ & $\begin{array}{l}1350 \mathrm{~W} \\
<5 \mathrm{~W} \\
13 \mathrm{~L} / \mathrm{min} \\
0.8 \mathrm{~L} / \mathrm{min} \\
0.74 \mathrm{~L} / \mathrm{min} \\
\text { Peak jumping } \\
1 \\
20.48 \mathrm{~ms}\end{array}$ \\
\hline
\end{tabular}

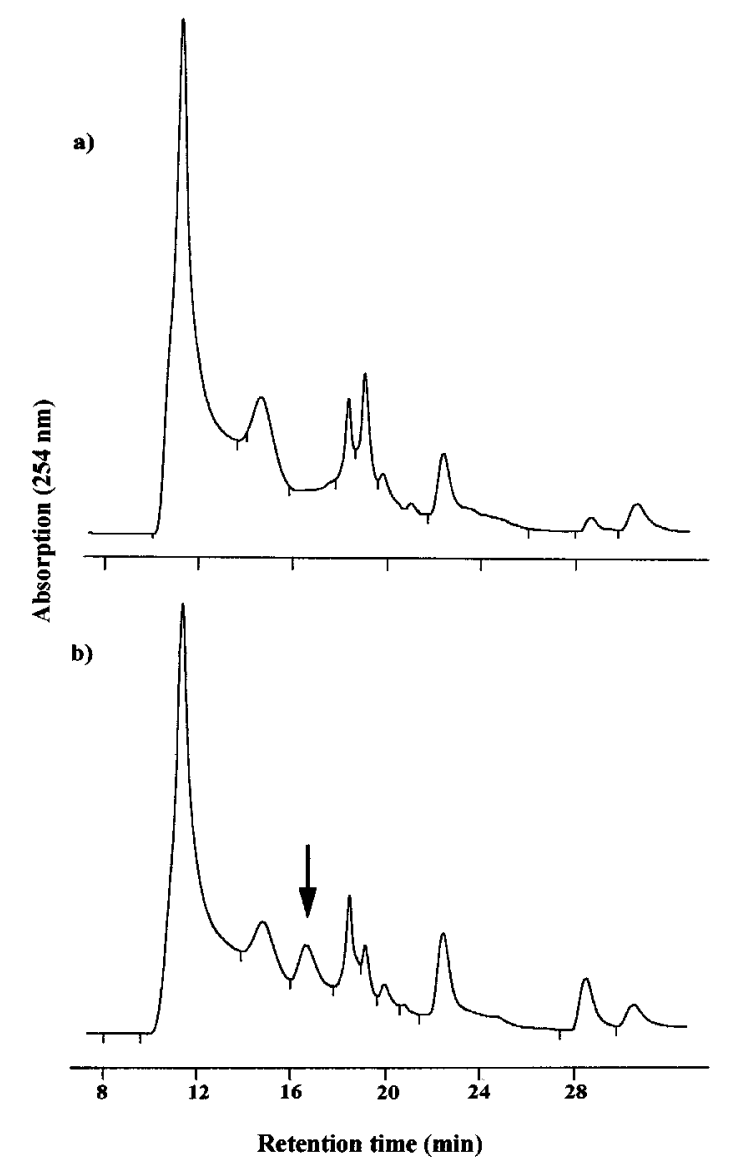

Figure 1. HPLC chromatograms of soluble extracts from tomato cell suspension cultures $5 \mathrm{~h}$ after $\mathrm{HM}$ incubation. a) control

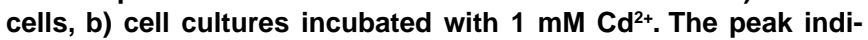
cated by an arrow corresponds to the HM-induced compound with an estimated molecular weight of approximately $13000 \mathrm{Da}$. Elution with $0.5 \mathrm{~mL} / \mathrm{min} 10 \mathrm{mM}$ ammonium acetate, $\mathrm{pH}$ 7. For Silene vulgaris cell suspension cultures see [7].

suspension cultures were analysed by electrospray ionisation mass spectrometry (ESI MS). Figure 3 shows the ESI mass spectrum of the HM-binding complex from a tomato cell suspension culture incubated with $0.01 \mathrm{mM} \mathrm{Cu}^{2+}$ and $\mathrm{Cd}^{2+}$. For tomato as well as for Silene vulgaris (data not shown) the HM-binding complexes can be clearly identified from the ICP MS measurements and the ESI mass spectra as a complex of HM ions and PC or desGly-PC molecules with the structure $(\gamma-\text { Glu-Cys })_{2}-$ Gly and $(\gamma-\text { Glu-Cys })_{2}$. PC molecules with $n>2$ are not found in the HM-PC-complexes after $24 \mathrm{~h}$ of HM exposition. The S-S-bonds observed in the mass spectrum seems to result from rearrangement reactions during the ionisation process or from the dissociation of the HM-PC-complexes. It is not clear, whether the desGly-PCmolecules are constituents of the HM-PC-complex with a function in HM-binding or if they are produced during the ionisation process.

In roots and leaves of water cultures of Silene vulgaris and tomato and in cell cultures exposed to equivalent concentrations of $\mathrm{Cu}^{2+}, \mathrm{Cd}^{2+}, \mathrm{Pb}^{2+}$ and $\mathrm{Zn}^{2+}$ only $\mathrm{Cu}-$ and $\mathrm{Cd}-$ $\mathrm{PC}$-complexes are detectable in the extracts, while $\mathrm{Pb}$ and $\mathrm{Zn}$ are bound to non-characterised lower molecular weight compounds. In vivo and in vitro saturation experiments with 


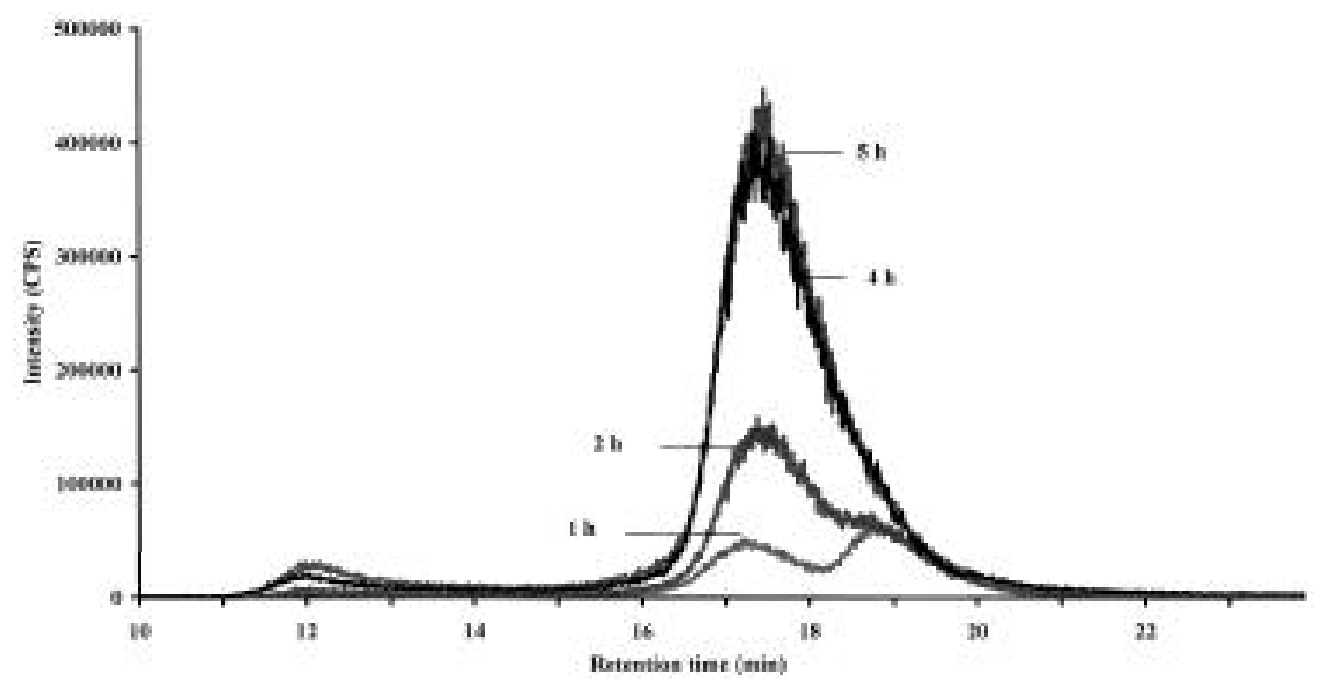

Figure 2. ICP-MS signals of the isotope ${ }^{114} \mathrm{Cd}$ in the soluble extracts of a tomato cell suspension culture incubated with $1 \mathrm{mM}$ Cd ${ }^{2+}$ for up to 5 hours. The peak with a retention time of approximately $17.5 \mathrm{~min}$ corresponds to the new synthesised compound indicated by an arrow in figure 1 . Elution with $0.5 \mathrm{~mL} / \mathrm{min} 10 \mathrm{mM}$ ammonium acetate, $\mathrm{pH}$ 7. The time difference between the UV and the ICP-MS detector was ca. $60 \mathrm{~s}$.

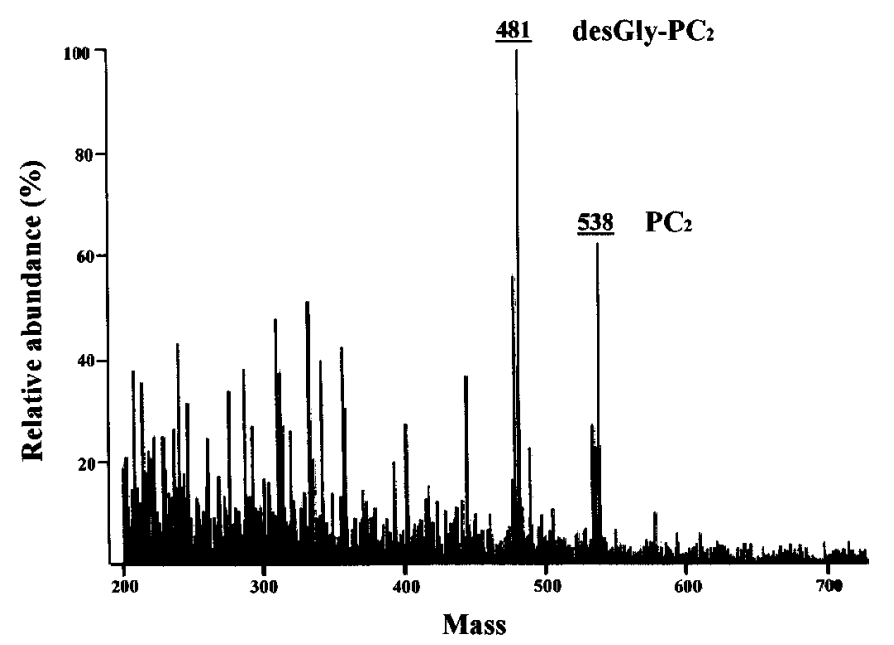

Figure 3. ESI mass spectrum of the purified HM-binding compound from a tomato cell suspension culture incubated with $0.01 \mathrm{mM} \mathrm{Cu}{ }^{2+}$ and $\mathrm{Cd}^{2+}$ for $24 \mathrm{~h}$. $\mathrm{PC}_{2}$ corresponds to $(\gamma-\text { Glu-Cys })_{2}-$ Gly; desGly-PC ${ }_{2}$ to $(\gamma-\text { Glu-Cys })_{2}$.

different concentrated $\mathrm{Cu}^{2+}$ - and $\mathrm{Cd}^{2+}$-solutions confirmed the higher binding stability of $\mathrm{Cu}^{2+}$ ions in the $\mathrm{PC}$-complexes in comparison to $\mathrm{Cd}^{2+}$ [9].

The amounts of HM ions in the soluble fractions of the samples were quantified from the HPLC-ICP MS experiments using HM-EDTA-solutions injected down-stream from the separation column as calibration standards. Certified reference materials for the speciation of low and high molecular weight HM-binding components in biological samples are not available. Therefore, a metallothionein-standard from rabbit liver (Sigma, St Louis, USA) was analysed to estimate the accuracy of the quantitative procedure. The recovery was determined to be better than $90 \%(n=5)$, despite memory effects on the column material. The total HM concentrations in the cell suspension cultures and whole plants were determined by direct nebulization into the ICP MS after microwave digestion. The comparison of the results of both quantitative measurements shows that only a minor part of the total HM concentration is present in a soluble form as well in cell suspension cultures (less then 5\%) as in whole plants (less then 25\%).

The investigation of HM tolerant Silene cucubalus, Minuartia verna, and Armeria maritima plants grown on a medieval copper mining region ("Saugrund", Eisleben, Germany) shows no formation of HM-PC-complexes in these plants. The HM ions in the soluble extracts are exclusively bound to lower molecular weight compounds. These results explain that PCs are not the major source of the high HM tolerance of these plants, although these plants are able to synthesise HM-PC-complexes as could be revealed in the water cultures. Using electron microscopic investigations combined with element-specific techniques it was confirmed that a couple of other mechanisms (e.g. HM excretion by specific glands or hydathodes in the leaves, binding of HM ions on proteins or silicates in an insoluble form, storage of $\mathrm{HM}$ ions in the intercellular spaces of the leaf parenchyma) is involved in the detoxification of high concentrations of essential and non-essential elements in the HM-tolerant plants of the copper mining region $[3,10]$.

\section{Conclusion}

The application of different concentrated HM solutions to cell suspension cultures and water cultures induces the synthesis of HM-PC-complexes with $\mathrm{Cu}$ and $\mathrm{Cd}$ ions as well in the HM tolerant Silene vulgaris as in the non-tolerant tomato under experimental conditions. $\mathrm{Zn}$ and $\mathrm{Pb}$ ions are exclusively bound to lower molecular weight substances in all investigated plant systems. HM-PC-complexes were not 
synthesised in HM tolerant Silene cucubalus plants grown on a medieval copper mining dump showing that PCs are not mainly responsible for the HM tolerance of these special phenotypes under natural conditions.

The high sensitivity of ICP MS combined with its ability to deal with a liquid eluent at typical chromatographic flow rates makes it an useful detector for multielemental speciation analysis in combination with a separation technique. An on-line coupled HPLC-ICP MS system was successfully used for the characterisation of HM-PC-complexes of different plant systems and for the investigation of the binding properties of these components under in vivo and in vitro conditions. However, there are a couple of limitations of this analytical system restricting the applicability of the technique especially for many biological samples. Low concentrations of sulphur or nitrogen which would be important for the qualitative or even quantitative analysis of amino acids or proteins as binding partners of trace elements are not detectable with ICP MS due to the high background signal on these elements. Furthermore, the determination of uncomplexed metal ions in the sample is hardly possible because they can be adsorbed to some extent on the size exclusion column material. Because certified reference materials for these types of HM-complexes are not available until now, the verification of quantitative results is complicated. Even considering the limitations of this system, the results obtained in the present study show the great potential of chromatographic techniques combined with an elemental detector. The careful optimisation of all parameters influ- encing the HPLC-ICP MS system and a accurate sample preparation lead to analytical results which allow more detailed insides into particular biological processes.

\section{Acknowledgements}

We thank Dr. Jürgen Schmidt from the Institute of Plant Biochemistry for the ESI-MS analyses.

\section{References}

1. Valle, B. L.; Ulmer, D. D. Ann. Rev. Biochem. 1972, 41, 91.

2. Ernst, W. H. O.; Verkleij, J. A. C.; Schat, H. Acta Bot. Neerl. 1992, 41, 229.

3. Neumann, D.; zur Nieden, U.; Lichtenberger, O.; Leopold, I. J. Plant. Physiol. 1995, 146, 704.

4. Krämer, U.; Cotter-Howells, J. D.; Charnock, J. M.; Baker, A. J. M.; Smith, J. A. C. Nature 1996, 379, 635.

5. Zenk, M. H. Gene 1996, 179, 21.

6. Grill, E.; Winnacker, E. L.; Zenk, M. H. Meth. Enzymol. 1991, $205,333$.

7. Rijstenbil, J. W.; Wijnholds, J. A. Marine Biol. 1996, 127, 45.

8. Vela, N. P.; Caruso, J. A. J. Anal. At. Spectrom. 1993, 8, 787.

9. Leopold, I.; Günther, D. Fresenius J. Anal. Chem. 1997, 359, 364.

10. Neumann, D. zur Nieden, U.; Schwieger, W.; Leopold, I.; Lichtenberger, O. J. Plant. Physiol. 1997, 151, 101. 